\title{
11. KÜNSTLERVERZEICHNIS
}

Dieses Verzeichnis listet in alphabetischer Reihenfolge alle Künstler auf, deren Graphiken, Karikaturen, Gemälde oder Skulpturen in der Arbeit beschrieben oder erwähnt werden. Auf die reiche Bibliographie, die es zu einigen berühmten Künstlern selbstverständlich gibt, wird hier nicht verwiesen. Nur teilweise leserliche Signaturen, auf die an entsprechender Stelle im Abbildungsverzeichnis verwiesen wird, finden sich hier nicht.

Als Basiswerke dienen in bezug auf die Karikaturisten:

Inventaire du fonds français (IFF), graveurs, après 1800. Paris, Bibliothèque nationale, cabinet des estampes, 1930-1985.

Osterwalder, Marcus, Dictionnaire des illustrateurs. 1800-1914, Paris 1983 (abgekürzt mit O I).

Ders., Dictionnaire des illustrateurs. 1890-1945. XX $\mathrm{X}^{\mathrm{e}}$ siècle. Première génération, Neuchâtel 1992 (abgekürzt mit O II).

Solo, Saint-Martin, Catherine, 5000 dessinateurs de presse et quelques supports. En France de Daumier à nos jours, Paris 1996 (abgekürzt mit Solo).

\section{Ergänzend wurde herangezogen:}

BÉNÉZIT, Emmanuel, Dictionnaire critique et documentaire des peintres, sculpteurs, dessinateurs et graveurs de tous les temps et de tous les pays. Nouvelle édition entièrement refondue, revue et corrigée, 10 Bde., Paris 1976 (abgekürzt mit BÉnézIr).

Hierauf wurde bei Karikaturisten dann zurückgegriffen, wenn in den beiden genannten Referenzwerken keine oder nur sehr geringe Angaben zu finden waren und zudem, um unklare Identitäten zu bestimmen.

Gliederung:

Künstler, die ausschließlich mit einem bestimmten Pseudonym signiert haben, nie aber mit ihrem bürgerlichen Namen, werden auch unter ihrem Hauptpseudonym in der Liste aufgeführt, mit Verweis auf den bürgerlichen Namen. Beispiel: Draner = Renard, Jules. Weitere, seltener verwendete Pseudonyme eines Künstlers werden, sofern mindestens eines der zitierten Bilder damit signiert ist, mit Verweis auf das Hauptpseudonym oder den bürgerlichen Namen in eckigen Klammern zusätzlich erwähnt. Beispiel: [Paf: vgl. Draner], [Saïd: vgl. Lévy, Alphonse].

Agard, Charles-Jean: 3.1.1866-1950. Französischer Maler.

BÉNÉZIT, Bd. 1, S. 52.

Asmodée = Machaux, Adrien: Reims, 1878-1962, Reims.

Signiert auch AM. Pseudonyme auch: Adrien Méria, Weal, Astaroth, Dayle, Héma, Ratichon.

Maler, Karikaturist, Plakatkünstler, Journalist, Redakteur und Schriftsteller. Gründet La Gifle (1908) und Le Cri (1909). Tätig u. a. für Le Rire, La Caricature, $L a$ Calotte (1907).

Solo, S. 676.
Bala d'Eche

Tätig für Le Triboulet.

SoLO, S.34.

Balluriau, Jean-Baptiste: 1860-1917, Paris. Signiert Paul Balluriau.

Autodidaktischer Illustrator und Karikaturist.

Tätig u. a. für Gil Blas illustré, Le Petit Bleu de Paris, L'Assiette au beurre, Le Rire.

Solo, S. 34f.

(Abb. 69, 70.) 
Baric, Jules: Sainte-Catherine-de-Fierbois, 1830-1905, Montmartre.

Pseudonyme: J. J. Antoine, Craïb, Julius

Altkind, Croïlo.

Karikaturist und Illustrator.

Tätig u. a. für Le Charivari.

Solo, S. 37f.

Barré, Raoul; Montreal 29.1.1874-1932.

Pseudonym auch: Varb.

Karikaturist und Cartoonist.

Tätig u.a. für Le Sifflet (1898).

Solo, S. 39; O II, S. 62.

Belloguet, André

Signiert auch: Belloquet.

Lithograph und Karikaturist.

Tätig u. a. für L'Éclipse, Le Grelot,

Le Cri-Cri.

Solo, S. 47.

Bernay, Paul: ?-1885.

Pseudonym auch: Candide.

Karikaturist, Lithograph, Silhouetten-

künstler, Bilderbuchillustrator.

Tätig u. a. für Le Grelot, La Lune rousse, Le Sifflet.

SoLo, S. 56.

Bertall = Arnoux, Charles-Albert de:

Paris, 1820-1882, Soyons.

Sein Künstlername stellt ein Anagramm aus seinem zweiten Vornamen dar.

Illustrator, Karikaturist, Plakatkünstler und Fotograf.

Solo, S. 56f.

Blass, (J.) = Douat, Albert: Bordeaux 1847-1892.

Signiert J. Blass.

Pseudonyme auch: Bourgevin, Gibet, Pouf, Spoer.

Illustrator und Karikaturist.

Tätig u. a. für Le Grelot, Le Triboulet, Le Courrier français, La Comédie politique,

Le Pilori, Le Figaro.

Gründet 1877 L'Éclair.

Solo, S. 65.

(Abb. 16, 54.)

Blondel: Möglicherweise identisch mit

Blondel, Carl-Albert: Stellt Anfang des

20. Jahrhunderts in Paris aus.

BÉNÉZIT, Bd.2, S. 91.

[Bobb: vgl. Morland, Valère]
Bonhomme, Jacques: Vermutlich ein Pseudonym, in keinem Referenzwerk verzeichnet.

Bréger, A. E.

Pseudonym auch: Tel.

Karikaturist und Plakatkünstler.

Tätig für Le Pèlerin (1913).

SoLo, S. 626.

(Abb.35.)

Brousset, B.

Tätig für Le Pèlerin.

SoLO, S. 82.

(Abb. 92.)

Cadel, Eugène: Paris, 1867-1940, Paris.

Maler, Karikaturist, Plakatgestalter.

Tätig u. a. für Le Rire, L'Assiette au beurre.

Solo, S. 90; O II, S. 208.

(Abb. 311.)

Caillebotte, Gustave: Paris,

19. 8.1848-21.2.1894, Gennevilliers.

Maler und Kunstmäzen.

BÉNÉZIT, Bd.2, S.447f.

Caran d'Ache = Poiré, Emmanuel: Moskau, 1858-1909, Paris.

Entstammt einer in Rußland ansässigen französischen Familie.

Sein Künstlername bedeutet auf russisch

»Bleistiftstummel .

Karikaturist, Autor von Bildergeschichten und Cartoons, Silhouettenkünstler.

1898 Mitbegründer von Psst...!

Tätig u. a. für L'Éclipse, La Caricature, L'Écho de Paris, Le Rire, L'Assiette au beurre.

Solo, S. 100-102.

Carrier, Ed

Karikaturist und Autor von Bildergeschichten.

Tätig u.a. für L'Assiette au beurre.

Solo, S. 109.

Cham = Comte de Noé, Amédée:

26.1.1818 oder 1819-Sept. 1879, Paris.

Karikaturist und Illustrator.

Nach 1870 u. a. tätig für Le Charivari.

Solo, S. 116; O I, 230.

(Abb. 5, 7, 9, 23, 24, 88, 89.)

[Chanteclair: vgl. Cohl, Émile]

Charvic

Solo, S. 124. 
Chevalier, Louis

Wahrscheinlich identisch mit Chevalier,

Louis Marie Jean-Baptiste.

Tätig u. a. für Le Sifflet (1898-1899).

BÉNÉZIT, Bd.2, S. 718; Solo, S. 129.

(Abb. 75.)

Chifflard, M. = Chifflart, Nicolas-François:

Saint-Omer, 21.2.1825-19.3.1901, Paris.

Maler und Graphiker.

Tätig u. a. für Le Monde illustré.

BÉNÉZIT, Bd. 2, S. 726; IFF, Bd. 4

S. 1526-1528; O I, S. 236.

Christian: Möglicherweise identisch mit

Christian, Paul Marie Bernard, der am

Salon von 1876 debütierte.

BÉNÉZIT, Bd.3, S.19.

(Abb.48.)

Clément, Jean: Wahrscheinlich identisch mit Clément, Jean-Jacques: Neapel, 15.1.1872-?

Maler und Graphiker.

Solo, S. 132; Bénézit, Bd. 3, S.61.

(Abb.312)

Clésinger, Jean, Baptiste-dit Auguste:

Besançon, 20.10.1814-6.1.1883, Paris.

Maler und Bildhauer.

BÉNÉZIT, Bd. 3, S. 68.

Cochin, Charles Nicolas le jeune: Paris,

1715-1790, Paris.

Maler und Stecher, Graphiker.

BÉnÉzit, Bd. 3, S.83f.

Cohl, Émile: Eigentlich Courtet, Émile:

Paris, 1857-1938, Villejuif.

Pseudonym für La Libre Parole illustrée:

Chanteclair.

Karikaturist und Pionier des französischen

Animationsfilms.

Seit 1883 Chefredakteur von La Nouvelle

Lune.

Tätig u. a. für Le Rire, Le Chat noir.

Solo, S. 135.

(Abb. 14, 45, 52.)

Couturier, Édouard: Paris, 1869-1935, Paris.

Karikaturist und Gestalter von Ansichts-

karten.

Tätig u. a. für Le Chambard socialiste, Le

Rire, La Feuille, Le Petit Bleu de Paris, Le

Sifflet, Le Fouet, Les Temps nouveaux, L'Assiette au beurre, Le Cri de Paris.

Solo, S. 144.

(Abb. 90.)
Dalou, Aimé-Jules: 31.12. 1838-15.4.1902.

Bildhauer.

BÉNÉZIT, Bd. 3, S. 334.

Darjou, Alfred: Paris, 1832-1874.

Karikaturist, Illustrator, Plakatgestalter.

Tätig u. a. für Le Charivari, La Lune,

L'Éclipse, Le Grelot.

SoLo, S.154.

(Abb.4.)

Daumier, Honoré: Marseille,

26.2.1808-11.2.1879, Valmondois.

Karikaturist und Maler.

Nach 1870 u. a. tätig für Le Charivari, Le

Peuple souverain, La Vie moderne, Le

Grelot.

Solo, S. 155-159; O I, 290 f.

Werkverzeichnisse: DELTEIL, SCHRENK.

(Abb.3.)

Debat-Ponson, Édouard-Bernard:

Toulouse, 25.4.1847-29.1.1913, Paris.

Maler.

BÉNÉZIT, Bd. 3, S. 405.

(Abb.72.)

Delannoy, Aristide: 31.7.1874-5.5.1911,

Paris.

Pseudonym auch: P. Sec.

Karikaturist und Graphiker.

Tätig u. a. für Gil Blas illustré, La Caricature, Le Cri de Paris, L'Assiette au beurre, Le Rire, Les Temps nouveaux (1905-1911), La Voix du peuple (1906), L'Humanité (1908),

Le Courrier français.

Solo, S. 164f.; O II, $287 \mathrm{f}$.

(Abb. 58.)

Draner $=$ Renard, Jules: Liège,

11.11.1833-1926, Paris.

Anm.: Nicht zu verwechseln mit dem gleichnamigen Schriftsteller Jules Renard (1864-1910).

Der Künstlername stellt ein Anagramm aus dem Nachnamen dar.

Pseudonym auch: Paf.

Illustrator und Karikaturist.

Tätig u. a. für Le Charivari, L'Éclipse, La

Caricature, Le Petit Bleu de Paris.

Solo, S. 189f.; O I, S. 324.

(Abb.6, 27.)

Duplessis, Joseph-Siffrède: Carpentiers, 22.9.1725-1.4.1802, Versailles.

BÉNÉZIT, Bd.4, S.33f. 
Edward, George: Sète (Hérault),

21.4.1866-?

Signiert auch George-Edward.

Illustrator und Karikaturist.

O II, S.352; BÉNÉZIT, Bd.4, S. 676.

Fau, Fernand: Poitiers, 1858-1919, Paris.

Pseudonyme: Poitevin, Fô, Phô.

Karikaturist, Maler, Autor von Bildergeschichten. Illustrator.

Tätig u. a. für Le Rire, Le Courrier français.

Solo, S. 219.

Fevre, D.

Wahrscheinlich identisch mit: de Feure,

Georges (eigentlich Georges Joseph von

Sluijters): Paris, 6.9.1878-26.11.1943,

Paris.

Maler und Graphiker holländischer Ab-

stammung.

Tätig u. a. für Le Courrier français.

BÉNÉzIT, Bd. 4, S.350; SOLO, S. 225.

Forain, Jean-Louis: Reims,

23.10.1852-11.7.1931, Paris.

Pseudonyme auch: For, A. Zut.

Karikaturist, Graphiker und Illustrator.

Mitbegründer des Anti-Dreyfus-Magazins

Psst!...

Tätig u. a. für L'Éclipse, La Journée, Le

Figaro, Gil Blas, L'Écho de Paris,

L'Assiette au beurre, Le Témoin.

Solo, S.231f.; O I, S.376f.

(Abb. 99.)

Gallo, Armand

Pseudonyme auch: Gallarmand, Larmand.

Tätig u. a. für L'Assiette au beurre.

Solo, S.250.

(Abb. 91.)

Gilbert-Martin, Charles: 26.8.1839-Juli

1905, Paris.

Karikaturist, Schriftsteller, Journalist.

Gründer von Le Don Quichotte.

O I, S. 428; Solo, S. $267 \mathrm{f}$.

Gill, André = Gosset de Guines, André:

Paris, 17.10.1840-1.oder 2.5. 1885.

Maler, Karikaturist und Journalist.

Gründer und Herausgeber von La Lune,

L'Éclipse, La Petite Lune.

O I, S. 429; Solo, S. 269f.

(Abb. 21, 22, 41, 42, 44.)
Girard, Eugène: Paris 1806-1881, Paris.

Karikaturist, Stecher, Porträtist, Kostüm-

bildner.

Tätig u.a. für La Caricature.

SoLo, S.271f.

Gottlob, Fernand: Paris, 1873-1935, Paris.

Karikaturist und Illustrator.

Tätig u. a. für Le Rire, Le Sifflet (1898),

L'Assiette au beurre, Pages folles, Gil Blas illustré. Solo, S.277.

(Abb. 36.)

Goussé, Henri: Gémozac, 1872-1914.

Karikaturist, Illustrator, Plakatkünstler.

Tätig u.a. für L'Assiette au beurre, Le Rire, Le Cri de Paris.

Solo, S. 280.

Grandjouan, Jules-Félix: Nantes,

22.12.1875-12.11.1968.

Karikaturist, Graphiker und Plakatkünstler.

Tätig u. a. für L'Assiette au beurre.

Solo, S. 283f.; O I, S. 445.

(Abb. 78, 79, 84, 84.)

Grandville, Jean-Ignace Gérard: Nancy, 1803-1847, Vanves.

Zeichner, Aquarellist, Lithograph, Buchillustrator und Karikaturist.

Tätig u.a. für La Caricature (1830-1839),

Le Charivari.

Solo, S.285f.

Grippa, A. = Lafosse, Georges: 1844-1880.

Karikaturist.

Zunächst überzeugter Republikaner, wendet sich dann aber den Monarchisten zu.

Tätig u.a. für Le Charivari, La Caricature, Le Triboulet.

Solo, S. 370.

(Abb. 39.)

Gumery, Adolphe: Paris, 1861-1918, Paris.

Maler, Karikaturist und Illustrator.

Tätig u. a. für Le Rire.

Solo, S. 300 .

Habert, Eugène: Paris, ?-August 1916,

Saint-Jean-le-Thomas.

Maler, Graphiker und Dekorationsmaler.

Tätig u. a. für Le Courrier français.

BÉNÉZIT, Bd.5, S. 339; Solo, S. 303.

(Abb.2.) 
Hadol, Paul: Rouen, 1835-1875, Paris.

Pseudonym auch: Job.

Karikaturist und Illustrator.

Tätig u.a. für Le Charivari, L'Éclipse, Almanach de l'Éclipse.

O I, S. 475.

Haenen, oder Haenens, Frédéric de.

Niederländischer Illustrator, zwischen 1882 und 1885 in Paris.

Tätig u. a. für L'lllustration.

IFF, Bd. 10, S. 95.

Hawkins, Louis Welden: Stuttgart, 1849-1910, Paris.

Maler und Graphiker. Gebürtiger Brite, aufgewachsen in Deutschland, seit 1895

naturalisierter französischer Staatsbürger.

Als Graphiker tätig für La Volonté.

BÉNÉZIT, Bd.6, S.814.

Heidbrinck, Oswald: 1860-5.3.1914, Paris.

Karikaturist, Illustrator und Plakatkünstler.

Tätig u. a. für Le Courrier français, Le

Rire, Le Charivari, Les Temps nouveaux,

L'Assiette au beurre.

Solo, S.310f.; O I, S. 486.

(Abb. 83, 96.)

Hellé = Laclôtre, André: Paris, März 1871-29.12.1945, Paris.

Maler, Theaterdekorateur, Kostümbildner, Illustrator und Karikaturist.

Tätig u. a. für Le Rire, La Caricature,

L'Assiette au beurre, Pages folles.

Solo, S.311f.; S. OII, $510 f$.

(Abb. 102.)

Hénault, Jules: 1859-?

Karikaturist und Graphiker.

Tätig u. a. für L'Assiette au beurre, Les

Temps nouveaux.

Solo, S.312.

(Abb. 26.)

Henriot = Maigrot, Henri: Toulouse, 1857-1933.

Pseudonym auch: Pif.

Karikaturist und Illustrator, ab 1890

Direktor von Le Charivari.

Tätig für Le Charivari, L'Événement, Le

Petit Bleu de Paris, Le Pèlerin.

Solo, S.313f.; O I, S. 492.
Hermann-Paul = Hermann, René-Georges

Paul: Paris 1874-Juli 1940.

Signiert auch: HP.

Karikaturist und Illustrator.

Tätig u. a. für Le Courrier français, Le

Rire, Le Cri de Paris, Le Petit Bleu de

Paris, Le Figaro, L'Assiette au beurre, Les

Temps nouveaux, Le Témoin.

Solo, S.317f.; O I, S. 496f.

Hope = Choubrac, Léon: Paris, 1847-1885

Paris.

Karikaturist, Illustrator und Plakatgestalter.

Tätig u. a. für L'Éclipse, Le Chat noir.

Solo, S. 322.

(Abb. 94.)

Ibels, Henri-Gabriel: Paris, 30.11.1867-

Februar 1936.

Maler, Illustrator, Karikaturist, Plakat-

künstler.

Gründet 1898 Le Sifflet.

Tätig u. a. für L'Écho de Paris, Le Cri de

Paris, L'Assiette au beurre, Le Rire, Le Gil

Blas.

Solo, S.331f.; O I, S. 523.

(Abb. 32, 64, 74.)

Isoré, Luis

Karikaturist.

Gründet 1882 La Halle aux charges.

SoLo, S.338.

Jankowski, Ch.-B.

Tätig u. a. für L'Assiette au beurre.

Solo, S. 342.

(Abb. 103.)

Jehannet $=$ Petitjean, Honoré: 1854-1929.

Pseudonym auch: Petit Jean.

Karikaturist und Graphiker.

Tätig u. a. für Le Rire, L'Assiette au beurre, Le Témoin, Les Temps nouveaux.

Solo, S. 510.

[Job: Vgl. Hadol, Paul]

Jossot, Gustave-Henri: Dijon, 1866-1951,

Algerien.

Maler, Karikaturist, Plakatkünstler, Illu-

strator und Autor.

Tätig u. a. für Le Rire, Le Cri de Paris,

L'Assiette au beurre, Les Temps nouveaux.

Solo, S. 355 . 
Jouve, Paul: 1878-1973.

Maler, Bildhauer, Tierzeichner, Illustrator, Karikaturist.

Tätig u. a. für L'Assiette au beurre.

Solo, S.360; O II, S. 577.

[Kab: Vgl. Morland, Valère]

Kauffmann, Peter Paul: Belfort, 8.7.1849-?

Illustrator und Karikaturist.

Tätig u. a. für Le Charivari, La Caricature. SoLo, S.363; O I, S. 546.

(Abb. 20)

Klenck, Paul: Basel, 1844-?

Pseudonyme auch: Kape, Tapdur, Filozel.

Illustrator und Karikaturist.

Tätig u. a. für Le Cri-Cri, Le Courrier

français.

SoLO, S. 366; BÉNÉZIT, Bd. 6, S. 241.

Kupka, František: Opocno, 1871-1957,

Puteaux.

Maler und Graphiker, Illustrator, Plakat-

künstler, Autor und Karikaturist.

SoLo, S. 367; BÉNÉzIT, Bd. 6, S.337-339.

(Abb.31.)

Ladreyt, Eugène: Sauzet, 1832-?

Karikaturist und karikaturistischer Bildhauer.

Gründet die Magazine: Guguess! (1870)

und Rigoletto (1871).

Tätig u.a. für Le Grelot, La Nouvelle Lune. SoLo, S. 369.

Lamouche, Eugène, 1864-?

Karikaturist.

Pseudonyme auch: E. Mille, Lek.

Tätig u. a. für $L e$ Courrier français, $L a$

Journée, L'Éclipse, La Silhouette, La

Caricature, Le Charivari.

SoLo, S. 372.

Launay $=$ Viellard, Fabien: Paris, 1877-1904, Paris.

Signiert oft: L. Pseudonym auch: Jacquet.

Karikaturist, Werbegraphiker und

Illustrator.

Tätig u. a. für Le Rire, L'Assiette au beurre. Solo, S. 377.
Léandre, Charles: 1862-1934, Paris.

Maler und Karikaturist.

Tätig u. a. für Le Rire, Gil Blas illustré, Le

Figaro, Le Cri de Paris, La Feuille, Le Petit

Bleu de Paris, L'Assiette au beurre, Le

Courrier français.

Solo, S.380f.

(Abb. 98.)

Lebasque, Henri: 25.9.1865-August 1937.

Maler und Graphiker.

BÉNÉZIT, Bd. 8, S.373f.

Lefebvre, Jules: Tournon,

14.3.1836-24.2.1911, Paris.

Porträtist und Genremaler.

BÉNÉZIT, Bd. 6, S. $534 \mathrm{f}$.

Lefman

Tätig für Le Grelot.

Solo, S. 385.

(Abb. 8.)

Legrand, Louis: 23.9.1863-12.6.1951.

Maler, Karikaturist und Illustrator.

Tätig u. a. für La Journée, Le Courrier français, Gil Blas illustré.

Solo, S.386; O I, S.605.

(Abb. 1.)

Lemaire:

Stecher, Anfang des 19. Jahrhunderts in Paris nachgewiesen.

Lemot, Valentin-Achille 1847-1909.

Signiert meist: A. Lemot.

Pseudonyme auch: Lilio (für Le Triboulet), Uzès. Manche Zeichnungen sind auch mit "U« signiert oder zeigen eine schwarze

Katze.

Karikaturist und Illustrator. Schüler von

André Gill.

Tätig u. a. für Le Courrier français,

L'Éclipse, Le Charivari, Le Triboulet, Le

Pélerin.

Solo, S. 388; IFF, Bd. 8, S. 495.

(Abb. 17, 33, 34, 37.)

Lepetit, Alfred: 8.6.1841-15.11.1909.

Signiert meist Alfred Le Petit. Pseudo-

nyme auch: Caporal, Le Grand, Zut.

Maler, Karikaturist, Chansonnier.

Künstlerische Leitung von La Charge

(1870-1871). Gründet Le Pétard

(1877-1879), Le Sans-Culotte (1878-1879),

La Charge (1888), L'Etrille (ab 1898). 
Tätig u. a. für L'Éclipse, Le Grelot, Le Charivari, La Nouvelle Lune, L'Assiette au beurre.

Solo, S.391f.; O I, S. 622.

(Abb. 18, 28, 50.)

Lévy, Alphonse: Marmoutier 1845-1918, Alger.

Pseudonyme auch Coco und Saïd.

Karikaturist, Cartoonzeichner, Illustrator und Plakatkünstler.

Tätig u. a. für L'Éclipse, La Caricature,

Le Rire.

SoLo, S. 396.

[Lilio: Vgl. Lemot]

Lix, Frédéric: Strasbourg 1830-1897, Paris.

Maler, Graphiker, Illustrator.

Tätig u.a. für Le Monde illustré.

Solo, S. $399 \mathrm{f}$.

Luce, Maximilien: Paris, 1858-1941, Paris.

Neoimpressionistischer Maler, Illustrator und Karikaturist.

Tätig u. a. für Le Père peinard, Le Chambard socialiste, Les Temps nouveaux, La

Feuille, La Voix du peuple.

SOLO, S. 405; BÉNÉZIT, Bd. 7, S. $7 \mathrm{f}$.

Lunel, Ferdinand: Paris, 1857-1938,

Boulogne-sur-Mer.

Karikaturist, Plakatkünstler, Schriftsteller.

Tätig u. a. für Le Courrier français, Le

Rire.

Solo, S. 406.

Luque, Manuel: Almeira, 1854-?

Spanischer Karikaturist, Pressezeichner und Illustrator.

Tätig u.a. für La Caricature.

Solo, S.407.

Maillet, Joseph oder Jean-Joseph: Paris, 1751-31.5. 1811, Paris.

Stecher.

BÉNÉZIT, Bd. 7, S. 75

Martial: Potémont, Adolphe Martial: Paris, 1828-1885, Paris.

Karikaturist und Graphiker.

Tätig u. a. für La Bataille.

Solo, S. 423

(Abb.111.)
Maurin, Charles: 1856-1914.

Karikaturist und Graphiker.

Tätig u. a. für Les Temps nouveaux, Almanach du Père peinard.

Solo, S. 428.

Métivet, Lucien: Paris, 1863-1932, Ver-

sailles.

Pseudonym auch: Luc.

Karikaturist.

Tätig u. a. für Le Rire, L'Assiette au beurre.

Solo, S. 434f.

Meyer, Henri: Mulhouse, 1844?

Pseudonym auch: Reyem.

Karikaturist und realistischer Illustrator.

Tätig u. a. für Le Sifflet.

Solo, S. 436.

(Abb.61, 66.)

Mirande, Henri: Levallois-Perret,

1877-1955, Paris.

Karikaturist und Maler.

Tätig u. a. für Le Rire, L'Assiette au beurre.

Solo, S. 442.

Mobb: In keinem Referenzwerk verzeichnet.

Moloch $=$ Colomb, Hector: Paris, 1849-1909, Paris.

Karikaturist und Werbegraphiker.

Tätig u. a. für L'Éclipse, Le Grelot, La

Caricature, La Silhouette, Le Chambard

socialiste, La Calotte, Le Rire, L'Assiette

au beurre.

Solo, S. 447.

(Abb. 47, 51, 53.)

Moreau-Nélaton, Adolphe Étienne

Auguste: 21.12.1859-25.4.1927.

Maler und Plakatkünstler.

BÉNÉZIT, Bd. 7, S. 530f.

Morin, Edmond: Le Havre, 1824-1882,

Sceaux.

Karikaturist, Pressezeichner und Illustrator.

Tätig u. a. für Le Monde illustré.

Solo, S.455.

(Abb. 46.)

Morland, Valère: 1846-?

Pseudonyme: Bobb, Lerory, Kab, Valio, Momus.

Karikaturist, Pressezeichner, Illustrator.

Tätig u. a. für Le Charivari, L'Éclipse, $L a$

Caricature, Le Triboulet, Le Pilori, La

Silhouette, La Comédie politique.

SOLO, S. 458; BÉNÉZIT, Bd. 7, S. 549. 
Ogé, Eugène

Karikaturist und Plakatkünstler.

SoLo, S. 476; BÉNÉZIT, Bd. 7, S. 788.

Ola = Olano, David

Karikaturist.

Tätig für Le Triboulet.

Solo, S. 477.

[Paf: Vgl. Draner]

Pépin, Édouard. Eigentlich: Guillaumin,

Édouard: 1842-?

Karikaturist.

Von 1871-1899 Titelkarikaturist bei Le

Grelot. Gibt 1899-1900 das Magazin Le

Fouet heraus.

Tätig u. a. für L'Éclipse, Le Charivari. O I, S. 817; SoLo, S. 503f.

(Abb. 29, 30.)

[Petit Pierre: Vgl. Steinlen, Théophile Alexandre]

Pezilla, Mario, ?-?.

Wahrscheinlich Italiener.

Karikaturist.

Tätig für Le Charivari, La Caricature, Le Rire.

Solo, S. 513.

(Abb. 87.)

Pilotell $=$ Pilotelle, Georges: Poitiers, 1845-1918, London.

Karikaturist und Illustrator. Engagierter

Communarde.

Solo, S.523f.

Pissarro, Camille: Saint-Thomas (Antillen), 10.7.1830-12.11.1903, Paris. Impressionistischer Maler, Graphiker und Illustrator.

BÉNÉZIT, Bd. 8, S.361-364.

Pissarro, Lucien: Paris, 1863-1944, Großbritannien. Sohn von Camille Pissarro.

Maler, Illustrator und Karikaturist.

Tätig u. a. für Le Courrier français, Le Père peinard.

Solo, S. 526; BÉNÉzIT Bd. 8, S. 364f.

Poncet, Paul:

Karikaturist.

Tätig für L'Assiette au beurre.

Solo, S. 532.

(Abb. 97.)

Quinsac, Paul-François: Bordeaux, 1858-?

Tätig für Le Courrier français.

Solo, S. 543 .
Raïeter, Gaston: 1881-?

Karikaturist.

Tätig u. a. für L'Assiette au beurre, $L a$ Barricade, Les Temps nouveaux, Le Courrier français.

Solo, S. 539.

(Abb.59.)

Rault, Louis-Armand: 1847-1903.

Maler und Graphiker.

BÉNÉzIT, Bd. 8, S. 618.

Redon, Georges: Paris, 1869-1943, Paris.

Karikaturist, Stecher, Graphiker, Illustrator und Blumenmaler.

Tätig u. a. für Le Rire, Le Petit Bleu de

Paris, L'Assiette au beurre.

Solo, S. 553; O II, S. 960.

(Abb.68.)

Renaux: In keinem Referenzwerk verzeichnet.

Robida, Albert: Compiègne, 1848-1926, Neuilly-sur-Seine.

Pseudonym auch: Roby.

Karikaturist, Illustrator, Autor, Silhouettenkünstler.

Gründer von La Caricature (1880-1914).

Solo, S. 568.

Roland

Identität unklar, möglicherweise identisch mit dem Blumenmaler Roland, der in der ersten Hälfte des 20. Jahrhunderts nachgewiesen ist. Dies würde das auffallende Blumendekor auf der zitierten Karikatur erklären.

SOLO, S. 571; BÉNÉZIT, Bd. 9, S. 54.

Roubille, Auguste: Paris, 1872-1955, Paris. Karikaturist, Graphiker, Plakatkünstler,

Bühnenmaler.

1903 Mitbegründer von Le Canard sauvage.

Tätig u. a. für Le Courrier français, Le Rire, Le Petit Bleu de Paris, Le Cri de Paris, L'Assiette au beurre, Les Temps nouveaux, Le Témoin (1906-1910).

Solo, S. 574.

Rysselberghe, Théodore von: 23 . oder 28.12.1862-13.12.1926.

Belgischer Maler und Graphiker.

BÉNÉZIT, Bd. 9, S. 206 f.

[Saïd: Vgl. Lévy, Alphonse]

Saint-Fourien

Tätig für La Calotte.

SoLo, S. 582. 
Sandy-Hook = Taboureau, Georges: Paris, 1879-1960, Paris.

Karikaturist und Illustrator.

Tätig u. a. für Le Ruy Blas.

Solo, S. 587.

(Abb. 107.)

Signac, Paul: Paris, 11.11.1863-15.8.1935, Paris.

Neo-impressionistischer Maler und Lithograph.

BÉNÉZIT, Bd. 9, S. 592-594.

Steinlen, Théophile Alexandre: Lausanne, 10.11.1859-14.12.1923, Paris.

Pseudonyme auch Petit Pierre, Jean

Caillou.

Maler, Graphiker, Plakatkünstler, Karikaturist.

Tätig u. a. für Le Courrier français, Le Père peinard, La Caricature, Gil Blas illustré, Le Chambard socialiste, Le Rire, Le Triboulet, La Feuille, Le Petit Bleu de Paris, La Voix du peuple, L'Assiette au beurre, Les Temps nouveaux, L'Humanité.

Solo, S. 618-620; BÉNÉZIT, Bd. 9, S. 810-812; O I, S. $1014 \mathrm{f}$.

(Abb. 77, 80, 93, 106, 108, 112-115.)

Stop $=$ Morel-Retz, Pierre Gabriel B. L.:

Dijon, 3.6.1825-5.9.1899, Dijon.

Rechtsanwalt, Maler, Karikaturist,

Illustrator.

Tätig u. a. für Le Charivari.

O I, S. 1020; Solo, S. 620 f.

(Abb. 10, 19.)

Sunyer y Miro, Joaquin (auch: Sunyer

Miro oder Sunyer): 1874 oder 1875-1956.

Maler und Graphiker.

BÉNÉzIT, Bd. 10, S. 12.

Tiret-Bognet, Georges: Saint-Servan, 1855-1935, Paris.

Pseudonyme auch Bec, G. Té Bé, Ti-Bec.

Karikaturist und Illustrator.

Tätig u. a. für La Caricature.

Solo, S.635.

(Abb. 86.)

Toulouse-Lautrec, Henri de. Eigentlich:

Toulouse-Lautrec-Monfa, Henri Marie

Raymond de: Albi, 1864-1901, Malromé.

Pseudonym auch: Treclau.

Prägender Maler der Belle Époque, Lithograph und Plakatkünstler.

Solo, S.639; BÉNÉZIT, Bd. 10, S. 245-247.
Tury, Henri:

Tätig für Le Triboulet.

Solo, S.647.

Vallotton, Félix: Lausanne, 1865-1925,

Paris.

Maler der Gruppe Nabis, Graphiker und Karikaturist.

Solo, S.653f.; BÉnÉzIT, Bd. 10, S.383-385.

Valveranne, Louis Joseph Marie Denis: 20.9.1870-12.4.1943.

Karikaturist, Autor von Bildergeschichten, Illustrator.

Pseudonyme auch: Denis Volx, Don Zed, Es, d'Es, Val, Valdès, Val d'Es, Montelli, Zed.

Tätig u. a. für Le Triboulet, Le Rire, Pages folles, Le Courrier français.

O II, S. 1202; Solo, S.654f.

(Abb. 95, 104.)

Vignola, Amédée

Beginnt seine Karriere als Karikaturist, wendet sich aber ab 1903 der Fotografie zu und gibt das Zeichnen auf.

Tätig u. a. für La Caricature, Le Charivari, Le Triboulet, Le Pilori.

Solo, S. 668

Willette, Adolphe Léon:

31.7.1857-4.2.1926, Paris.

Pseudonyme auch: Bébé, Cémoi, Louison, Nox, Pierrot, Vendredi.

Graphiker und Karikaturist.

Gründet Le Pierrot (1888-1891), La Vache enragée (1896-1897), Le Pied de nez

(1901), Les Humoristes (mit Steinlen, 1901).

Tätig u. a. für Le Figaro, Le Charivari, Le

Triboulet, Le Courrier français, L'Écho de Paris, La Libre Parole illustrée, Le Rire,

L'Assiette au beurre, Les Temps nouveaux

(1907).

O I, S. 1132; Solo, S.681f.

(Abb. 11, 101, 105.)

Wuyts, A. G, ?-?.

Tätig u. a. für Le Libertaire.

Solo, S. 684.

(Abb. 76.) 
